
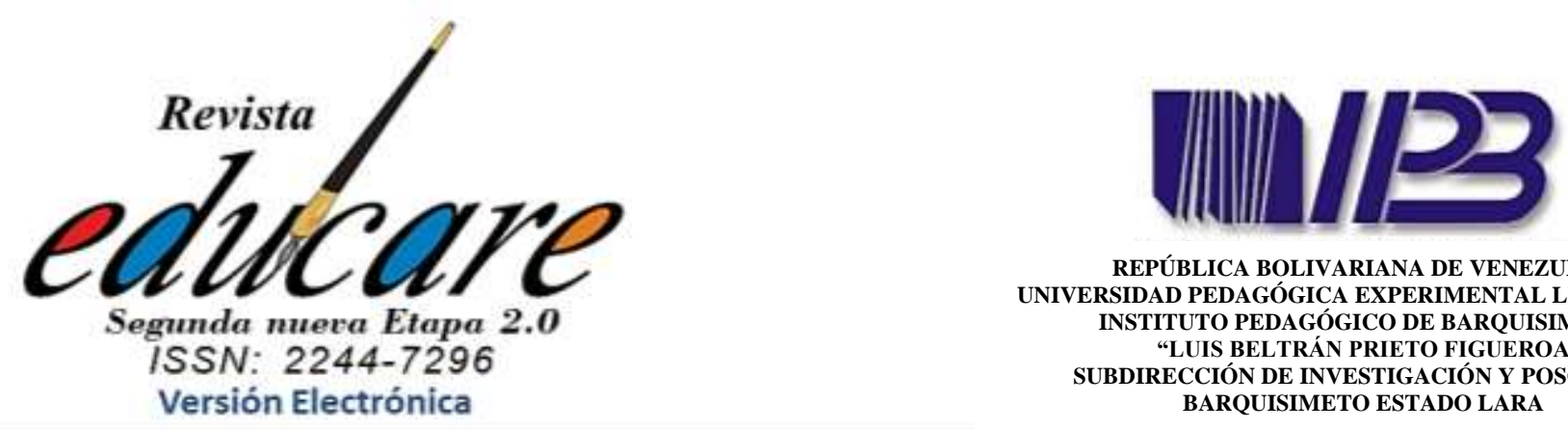

REPÚBLICA BOLIVARIANA DE VENEZUELA UNIVERSIDAD PEDAGÓGICA EXPERIMENTAL LIBERTADOR INSTITUTO PEDAGÓGICO DE BARQUISIMETO "LUIS BELTRÁN PRIETO FIGUEROA

SUBDIRECCIÓN DE INVESTIGACIÓN Y POSGRADO BARQUISIMETO ESTADO LARA

Norelvis Saturnini

ORCID: https://orcid.org/0000-0001-6420-9354

UNIVERSIDAD PEDAGÓGICA EXPERIMENTAL LIBERTADOR (UPEL-IPB)

Doctora en Ciencia de la Educación, Magister en Gerencia Educacional, Licenciada en educación mención Educación Especial, Secretaria encargada del instituto Pedagógico de Barquisimeto. E-mail; norelvissaturnini@hotmail,com

\section{FORMACIÓN TECNOLÓGICA DE HABILIDADES ADAPTATIVAS SOCIALES EN ESTUDIANTES DE EDUCACIÓN ESPECIAL}

\author{
TECHNOLOGICAL TRAINING OF SOCIAL \\ ADAPTIVE SKILLS IN SPECIAL EDUCATION \\ STUDENTS
}

Recibido:

5-12-2019

Aceptado:

16-02-2020 

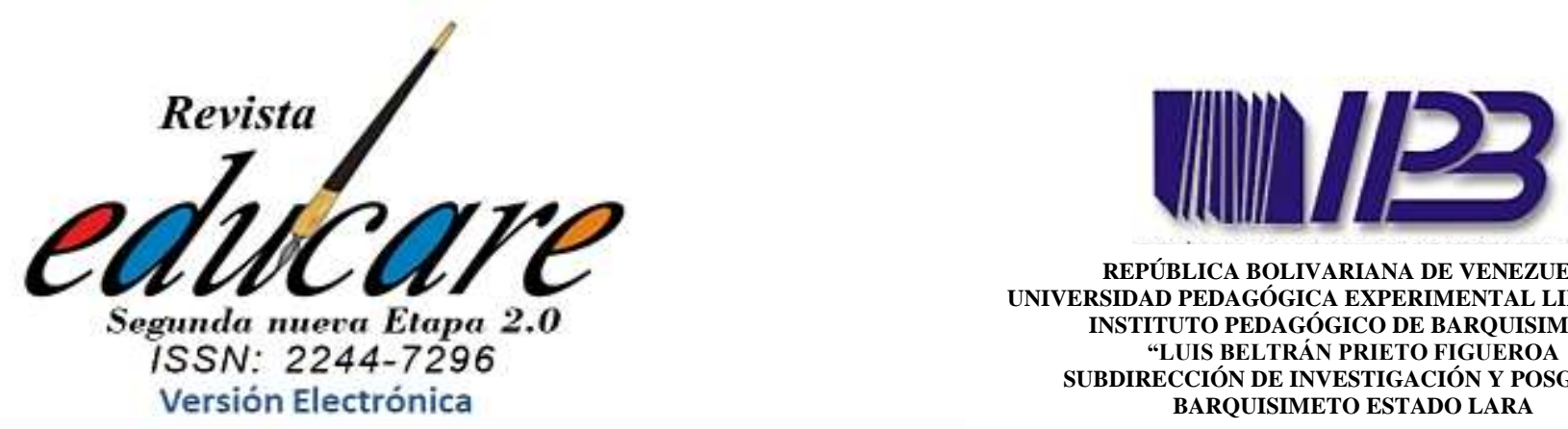

REPÚBLICA BOLIVARIANA DE VENEZUELA UNIVERSIDAD PEDAGÓGICA EXPERIMENTAL LIBERTADOR INSTITUTO PEDAGÓGICO DE BARQUISIMETO "LUIS BELTRÁN PRIETO FIGUEROA SUBDIRECCIÓN DE INVESTIGACIÓN Y POSGRADO BARQUISIMETO ESTADO LARA

\title{
Resume
}

El propósito de este ensayo es reflexionar acerca de la formación tecnológica de habilidades adaptativas sociales en estudiantes de educación especial. En esta idea, se presenta el acopio de la información a través de la revisión documental de algunos esquemas teóricos relacionados con los constructos de estudio a partir de la enseñanza especial y se asumen las Tecnologías de Información y Comunicación (TIC) como elementos transformadores de los flujos de información, capitales y saberes a partir de los cuales se está experimentando un desarrollo vertiginoso que de la sociedad. Se concluyó, que el acceso a los recursos tecnológicos permite la innovación en la praxis formativa que reconoce el valor de todas las ideas creativas, ajustadas a las necesidades pedagógicas para que el desarrollo de las habilidades adaptativas sociales, sean lo más efectiva posible, destinadas a la alfabetización, apropiación y uso de las tecnologías en todas sus manifestaciones.

TECHNOLOGICAL TRAINING

OF SOCIAL ADAPTIVE SKILLS IN SPECIAL EDUCATION STUDENTS

Descriptores: TIC, formación tecnológica, habilidades adaptativas sociales, educación especial.

\begin{abstract}
The purpose of this essay is to reflect on the technological training of social adaptive skills in special education students. In this idea, the collection of information is presented through the documentary review of some theoretical schemes related to the study constructs from special education and Information and Communication Technologies (ICT) are assumed as transforming elements of the information flows, capitals and knowledge from which a dizzying development is being experienced than that of society. It was concluded that access to technological resources allows innovation in training praxis that recognizes the value of all creative ideas, adjusted to pedagogical needs so that the development of social adaptive skills is as effective as possible, aimed at literacy, appropriation and use of technologies in all its manifestations.
\end{abstract}

Keywords: TICs, technological training, social adaptive skills, special education 


\section{SITUACIÓN DE INTERÉS}

Se vive una época donde autoridades ministeriales, docentes, representantes y activistas involucrados en el sistema educativo, debaten temas sobre la importancia que tienen los derechos de igualdad, desde los principios de normalización, modernización y democratización que constituyen la calidad de la educación venezolana, cuando a la población con necesidades educativas especiales se trata.

La constante discusión de estos principios, suelen ser trillados e improductivas cuando entre profesionales de la enseñanza especial se realiza un ejercicio de reflexión sobre la base del funcionamiento efectivo de las instituciones educativas de la modalidad de Educación Especial y la praxis del docente de aula ante la integración del estudiante con retardo mental en los procesos sociales.

Desde esta connotación educativa, el aprendizaje virtual debe estar orientada al desarrollo integral de los estudiantes de educación especial, lo que contribuirá al desarrollo de las facultades para adquirir, así como construir los conocimientos pertinentes, potenciar sus capacidades con las actitudes positivas para la incorporación de las Tecnologías de Información y Comunicación en sus actividades (TIC) cotidianas durante su formación pedagógica para que ejerza plenamente sus capacidades humanas, generando condiciones que garanticen su participación activa y estimulando sus iniciativas para desplegar aprendizajes tecnoeducativos. Los procesos de enseñanza-aprendizaje sobre todo, porque no cabe la menor duda, que una gestión tecnológica incrementó de forma sustancial la capacidad de reacción para incorporar nuevos conocimientos y avances tecnológicos emergentes de la globalización.

De este modo, el propósito de este ensayo es reflexionar acerca de la formación tecnológica de habilidades adaptativas sociales en estudiantes de educación especial. En esta idea, se presenta el acopio de la información a través de la revisión documental de algunos esquemas teóricos relacionados con los constructos de estudio.

\section{ANÁLISIS ARGUMENTATIVO}

Desde el principio de la humanidad han existido personas con deficiencias mentales, aunque el estudio sistemático de esta disciplina no comienza hasta el siglo XLX con los 
aportes de estudiosos dedicados a la atención de esta población. A lo largo del tiempo, se han utilizado diferentes calificativos que destacan algún tipo de diferencia psíquica o física a las de su grupo etario social o educativo, expresiones como: minusvalía, discapacidad psíquica, débil mental, retraso mental, oligofrenia entre otras, para referirse al mismo grupo.

Desde una perspectiva interdisciplinaria, se asume que el nivel de funcionamiento de una persona con necesidades educativas especiales, no está condicionada por las capacidades cognitivas representada por un valor dado en cualquiera de las escalas de inteligencia estandarizadas, a través de criterios establecidos que abordan únicamente tres aspectos, como la edad cronológica, la capacidad intelectual y la deficiencia en la capacidad adaptativa. En este ámbito formativo, la Educación Especial, representa una modalidad educativa según la Ley Orgánica de Educación (2009), y, de acuerdo a la conceptualización y políticas de Educación Especial (Ministerio de Educación, 1997):

Se rige por los mismos principios y fines de la educación general y representa la opción político-educativa para la población con necesidades educativas especiales entre ellas, las personas con retardo mental. La educación es un derecho colectivo e irrenunciable de los venezolanos sin más limitaciones que las derivadas de la vocación y de las aptitudes, garantizando la gratuidad de la enseñanza y el reconocimiento de la educación como un derecho ciudadano y un deber del Estado, el cual debe garantizar que las personas con retardo mental como ciudadanos, se beneficien de este derecho social (p. 42).

De acuerdo con estos principios, la educación especial en Venezuela, se define por su carácter social-humanista configurada por sustentos filosóficos centrados en la importancia del ser capaz de desarrollarse, en función a sus potencialidades sin excluir el reconocimiento de sus limitaciones físicas y mentales desde la perspectiva pedagógica, bajo una disciplina holística en su modelo de atención que incluye, no solo al estudiante, sino a su entorno desde tempranas edades con la intención de integrarles efectivamente a los campos socio-familiar y sociolaboral.

También, la Educación Especial, está constituida por un conjunto de apoyos técnicos y teóricos que orientan los procesos pedagógicos en el modelo de atención, con el objetivo de compensar la deficiencia que afecta significativamente el área de desarrollo cognitiva y las habilidades adaptativas con actitudes y aptitudes que favorezcan su autonomía e independencia dentro del sistema social que pertenecen. Mateos (2008) sostiene que: 
La educación especial debe verse en función de las acciones que se articulan, de la transformación, del cambio, de la reducción de la diferencia, de la adquisición de una competencia inexistente o limitada previamente y de cómo podemos intervenir para que el contexto nos favorezca a todos, sin pretender la normalidad de la persona que, por alguna causa, consideramos fuera de ésta por presentar algún rasgo distintivo (p.6).

De ahí que las prioridades existentes por medio del establecimiento de finalidades y objetivos adecuados, deben estar dirigidas a garantizar los derechos de atención educativa especializada de los estudiantes, entre ellos, los adultos, que requieren una formación pedagógica que los habilite laboralmente para desarrollar su autonomía.

De tal manera, la vinculación del acto educativo en el contexto comunitario constituye la formación no solo de los estudiantes con retardo mental sino al resto de los actores sociales. Esto a su vez habilita al estudiante para enfrentar los cambios en la dinámica social con las actividades culturales, deportivas, religiosas, laborales propias de los espacios donde el estudiante se desenvuelve y que favorecen el desarrollo progresivo de las destrezas necesarias para desenvolverse con otros. De acuerdo a Ramírez (2010) las habilidades adaptativas sociales se:

Manifiestan en una conducta social apropiada, así como a rechazar las manifestaciones conductuales inapropiadas. La conducta social apropiada incluye cómo hacer amistades, mostrar aprecio, sonreír, cooperar con los demás, ser honesto, entre las conductas sociales inapropiadas se encuentran los celos, la competitividad, mostrar conductas públicas de carácter sexual, entre otras (p.13).

En el ámbito pedagógico estas conductas deben ser compensadas a través de estrategias educacionales individualizadas en correspondencia a la necesidad de cada estudiante, de tal manera que los docentes puedan satisfacer los objetivos planteados por la institución.

Existen varios tipos de habilidades adaptativas, de acuerdo a Ramírez (ob.cit., p.13) entre las que se destacan la autodirección, salud, conocimiento funcional, tiempo libre y trabajo, todas ellas consideradas habilidades adaptativas sociales que permiten a la persona con necesidades especiales su incorporación eficaz a la sociedad.

En este sentido, las personas con necesidades especiales que según Brennan (1988), puede ser identificada como una condición "física, sensorial e intelectual, emocional o social o cualquier combinación de éstas, que afecta el aprendizaje" (p. 108). Por lo tanto, las habilidades adaptativas, hace referencia al desarrollo de potencialidades funcionales que no 
requiera que realicen operaciones cognitivas complejas, más allá de las requeridas por las situaciones, tareas o instrucciones sencillas que le permite interactuar en entornos de integración socio-familiar o socio-laboral.

Es importante destacar, que cuando una persona tiene una condición bien sea de tipo orgánico donde está comprometida el área cognitiva, puede desarrollar algunas de las habilidades adaptativas que se manifiestan en el aprendizaje, la conducta y el auto cuidado, las cuales pueden ser compensadas a partir un abordaje pedagógico apropiado. Lo que significa la posibilidad de integrarse a la vida en sociedad y también de participar como miembro productivo dentro del campo laboral.

En consecuencia, el desarrollo de habilidades adaptativas requiere el compromiso de potenciar ciertas destrezas relacionadas con (a) la organización en relación con saber actuar; (b) la planificación que le permita saber conocer los contenidos; (c) la ejecución de la praxis de aula que conlleva saber hacer; y (d) la evaluación de su gestión pedagógica que requiere saber pensar para reflexionar las adaptaciones curriculares (contenido, objetivos y metodología) acorde a las necesidades individuales y colectiva de la población con necesidades educativas especiales que le permitan su integración social al medio circundante.

La formación de los educandos en la modalidad de educación especial, los cuales requieren de una praxis didáctica, de los métodos pedagógicos aplicados a las personas con necesidades educativas especiales, ante la posibilidad de construir conocimientos significativos a partir de estrategias didácticas innovadoras. En este contexto educativo Castells (1997), describe que en todos los ámbitos en el siglo XXI, los cambios tecnológicos demanda la formación integral de los estudiantes con necesidades educativas especiales para ser habilitados socialmente en el acceso a la información y comunicación.

Las Tecnologías de Información y Comunicación (TIC), son conceptualizadas por el Programa de las Naciones Unidas para el Desarrollo (2002), como parte del conjunto de elementos transformadores que han traído como consecuencia la globalización de los flujos de información, capitales y saberes, a partir de los cuales se está experimentando un desarrollo vertiginoso que ha transformado todos los ámbitos de la sociedad, y por supuesto, no es una excepción.

En efecto, la influencia que las TIC tienen sobre las transformaciones socioeducativas de la modalidad de Educación Especial, en las cuales las concepciones de información y 
conocimiento, tienen un campo que van desde lo filosófico, hasta lo disciplinar específico, que constituyen la epistemología relacionada con estas tecnologías y su relación con las ciencias sociales

En este particular Vizer (2003), describe a las TIC, como un conjunto heterogéneo de técnicas, sistemas de aparatos electrónicos, máquinas "inteligentes", redes tecnológicas, programas informáticos, usos socio-técnicos y culturales que se hallan en pleno crecimiento exponencial, que se encuentran aún en una etapa primaria de búsqueda de marcos teóricos de interpretación que les sirvan de articulación con las acciones de enseñanza que conlleven a adquirir las habilidades adaptativas socializadoras.

Por esta razón, las instituciones de Educación Especial, deben considerar a las TIC, como medios de formación adecuados, que involucren las estrategias didácticas de enseñanza, para proporcionar o facilitar al estudiante con necesidades educativas especiales, la posibilidad de una alfabetización, uso y aprovechamiento de estos recursos para desarrollar las habilidades adaptativas socializadoras en esta modalidad formativa.

En correspondencia, existe una propuesta curricular del Ministerio del Poder Popular para la Educación (2007) que establece en el Diseño Curricular del Sistema Educativo Bolivariano las TIC como eje integrador. La incorporación de las TIC en los espacios y procesos educativos, contribuye al desarrollo de potencialidades para que incidan en la forma de enseñar del docente y aprender del estudiante con necesidades especiales.

En esta línea de pensamiento De Pablo (2002), hace referencia al rol de la tecnología, la cual se ha utilizado en todos los niveles, modalidades y formas del sistema educativo, con el empleo de aparatos, máquinas o herramientas que facilitan a través de la combinación de códigos para convertir la información en forma rápida, precisa y objetiva. El uso de las TIC está cada vez más generalizado en el ámbito educativo, familiar y social, lo que ofrece oportunidades para que las personas con necesidades especiales conozcan y utilicen las herramientas tecnológicas, tanto en el ámbito familiar, educativo y social.

Las TIC, son medios o herramientas innovadores que en opinión de Inciarte (2007), permiten formas de interacción para atender al aprendiz en las diferentes etapas de la vida y de los niveles educativos virtuales. Según Cabero (1996), la educación virtual, alude a la intangibilidad de la acción educativa, debido a que la materia prima es la información en 
diferentes formas y codificaciones tangible como un libro, por cuanto están contenidas en forma virtual.

En definitiva, las TIC en la modalidad de educación especial, ayudan a aumentar la capacidad de almacenamiento y de procesamiento de la información, mejoran la memoria semántica, relacionada con el significado de las palabras; además, centran la atención, posibilitan una mejor comprensión de lo abstracto, mejoran la generalización y el mantenimiento del aprendizaje; también, refuerzan la visión y/o la audición, así como la coordinación viso-motriz, corrigen trastornos importantes del lenguaje, fomentan la iniciativa para comenzar actividades y la constancia para realizar tareas menos motivadoras, favorecen la reflexión, optimizan la organización temporal, entre otros aspectos que favorecen la acción pedagógica.

\section{CONSIDERACIONES FINALES}

En respuesta al propósito de este ensayo acerca a la formación tecnológica de habilidades adaptativas sociales en estudiantes de educación especial, una vez hecha la revisión de los planteamientos teóricos que fundamentan las reflexiones como un todo integrado, a partir de los planteamientos teóricos, se infiere que las TIC requiere un modelo educativo que desarrollen las potencialidades funcionales de operaciones cognitivas requeridas en entornos de integración socio-familiar o socio-laboral.

En la sociedad del conocimiento, el uso de las TIC, no sólo requiere del conocimiento necesario por parte del docente para el acceso a programas y servicios, sino también, exige de su habilidad para incorporar en su praxis pedagógicas, el uso de estas tecnologías para mejoran la memoria semántica, centrar la atención, comprensión generalización y mantenimiento del aprendizaje de estudiantes con necesidades educativas especiales en un intento por potenciar una educación integral de calidad.

La importancia de la utilización de las TIC, en la modalidad de educación especial, tiene que ver con situar estas tecnologías como instrumento pedagógico destinado a potenciar las habilidades adaptativas socializadoras. Además de ser una herramienta capaz de mediar ámbitos de enseñanza-aprendizaje alternativos destinados a implementar alternativas didáctica que respondas a las necesidades, motivaciones e intereses de los estudiantes y se convierta en 
oportunidades para contrarrestar alguna de las dificultades derivadas de sus limitaciones biológicas, psicológicas y sociales.

Sin embargo, en Venezuela el uso de las TIC en la modalidad de educación especial es limitado debido a la falta de formación de sus docentes, así como de la dotación de recursos tecnológicos para fines pedagógicos. También, queda aún por determinar, si la integración de las Tecnologías de la Comunicación y la Información en ese ámbito es apropiada para las características de la población estudiantil con necesidades especiales.

Teniendo en cuenta todos estos puntos, los estudiantes con necesidades de atención especial - por lo general- es una población que presenta mayores dificultades para acceder a los aprendizajes, debido a la diversidad que presentan y no son contempladas en su totalidad en el currículo de educación especial. Entre las características personales y las actuaciones que recibe de su entorno educativo, no hay claridad en las estrategias de la intervención pedagógica particular a los requerimientos, lo que limita las posibilidades de apoyo y uso de los recursos tecnológicos que faciliten el acceso y/o adaptaciones para lograr los objetivos programáticos en la medida en que éstos sean necesarios.

El correspondencia, el uso de las TIC, en otros países, es un hecho consolidado, y la competencia digital es ya un indicador de calidad en el desarrollo escolar y social, es necesario que también se ofrezca a los estudiantes de la modalidad de educación especial, el acceso de las tecnologías como una respuesta educativa destinada a apoyar el desarrollo de las habilidades adaptativas en pro de alcanzar los objetivos educativos de integración de esta población estudiantil a la sociedad, acorde con las oportunidades de cada persona que tenga una condición de atención especial.

Finalmente, se concluyó que el acceso a los recursos tecnológicos permite la innovación en la praxis formativa que reconoce el valor de todas las ideas creativas, ajustadas a las necesidades pedagógicas para que el desarrollo de las habilidades adaptativas sociales, sean lo más efectiva posible, destinadas a la alfabetización, apropiación y uso de las tecnologías en todas sus manifestaciones en la modalidad de Educación Especial, para dar un salto cualitativo en los modos de relacionarse en los entornos digitales del ciberespacio, lo cual permite la incursión exitosa de los estudiantes con necesidades especiales. 


\section{REFERENCIAS}

Cabero, J. (1996). Nuevas tecnologías, comunicación y educación. Revista electrónica de tecnología educativa.

Castells, M. (1997). La era de la Información, Economía, Sociedad y Cultura. Vol.1: La Sociedad en Red. Madrid: Alianza Editorial.

De Pablo, J. (2002). Juan de Pablos Pons: La Tecnología Educativa en el Marco de la Sociedad de la Información (Prologo). Revista Fuentes. España.

Inciarte, M. (2007). Diseño Instruccional para Educación a Distancia. Sistema de Educación a Distancia Universidad del Zulia.

Ley Orgánica de Educación (2009). Gaceta Oficial de la República Bolivariana de Venezuela $\mathrm{N}^{\circ}$ 5.929, Extraordinario del 15 de agosto de 2009. Caracas, Venezuela.

Mateos, G. (2008). Educación Especial. [Documento en Línea] Disponible en: http://www.redalyc.org/pdf/802/80210101.pdf [Consulta, 2017 septiembre 11].

Ministerio de Educación (1997). Conceptualización y Política de la atención educativa integral de las personas con retardo mental. Caracas: Autor.

Ministerio de Poder Popular para la Educación (2007). Diseño Curricular del Sistema Educativo Bolivariano. Caracas: MPPE-CENAMEC.

Programa de las Naciones Unidas para el Desarrollo. (2002). Informe sobre Desarrollo Humano en Venezuela: Las tecnologías de la información y la comunicación al servicio del desarrollo. Disponible en: http://www.pnud.org.ve /idhn_2002/cap-6.pdf [Consulta 2016, octubre 16].

Ramírez, E. (2010). La formación laboral de los alumnos con retraso mental desde una nueva concepción pedagógica. Cuba: Pueblo y Educación.

Vizer, E. (2003). Las tecnologías de información y comunicación (TIC) y el crecimiento del capital social. Buenos Aires. 